\title{
Highly-Sensitive Multiplexed in vivo Imaging Using PEGylated Upconversion Nanoparticles
}

\author{
Liang Cheng ${ }^{1}$, Kai Yang ${ }^{1}$, Shuai Zhang ${ }^{1}$, Mingwang Shao ${ }^{1}(\bowtie)$, Shuittong Lee ${ }^{2}$, and Zhuang Liu ${ }^{1}(\bowtie)$ \\ ${ }^{1}$ Jiangsu Key Laboratory for Carbon-Based Functional Materials and Devices, Institute of Functional Nano and Soft Materials, Soochow \\ University, Suzhou, Jiangsu 215123, China \\ ${ }^{2}$ Center of Super-Diamond and Advanced Films (COSDAF) and Department of Physics and Materials Science, City University of Hong \\ Kong, Hong Kong, China \\ Received: 17 July 2010 / Revised: 18 August 2010 / Accepted: 22 August 2010 \\ (C) The Author(s) 2010. This article is published with open access at Springerlink.com
}

\begin{abstract}
Lanthanide-based upconversion nanoparticles (UCNPs) have been widely explored in various fields, including optical imaging, in recent years. Although earlier work has shown that UCNPs with different lanthanide $\left(\operatorname{Ln}^{3+}\right)$ dopants exhibit various colors, multicolor — especially in vivo multiplexed biomedical imaging — using UCNPs has rarely been reported. In this work, we synthesize a series of UCNPs with different emission colors and functionalize them with an amphiphilic polymer to confer water solubility. Multicolor in vivo upconversion luminescence (UCL) imaging is demonstrated by imaging subcutaneously injected UCNPs and applied in multiplexed in vivo lymph node mapping. We also use UCNPs for multicolor cancer cell labeling and realize in vivo cell tracking by UCL imaging. Moreover, for the first time we compare the in vivo imaging sensitivity of quantum dot (QD)-based fluorescence imaging and UCNP-based UCL imaging side by side, and find the in vivo detection limit of UCNPs to be at least one order of magnitude lower than that of QDs in our current non-optimized imaging system. Our data suggest that, by virtue of their unique optical properties, UCNPs have great potential for use in highly-sensitive multiplexed biomedical imaging.
\end{abstract}

\section{KEYWORDS}

Upconversion nanoparticles, multicolor imaging, lymphatic mapping, cell tracking, sensitive imaging

\section{Introduction}

There has been intense interest in the development of highly luminescent nanomaterials for multicolor, multiplexed molecular imaging [1-4]. Traditional fluorescence imaging based on organic fluorescent dyes or inorganic quantum dots (QDs) is being widely used for multicolor imaging in vitro and in vivo [5]. However, the background autofluorescence in tra- ditional fluorescence imaging techniques significantly limits the imaging sensitivity, especially for in vivo imaging in which the tissues and food residues exhibit strong background fluorescence [6]. In the past few years, studies on upconversion nanoparticles (UCNPs), usually containing lanthanide ions $\left(\mathrm{Ln}^{3+}\right)$, have grown rapidly owing to their wide applications in solidstate lasers, three-dimensional flat-panel displays, low-intensity infrared (IR) imaging, bioprobes, and

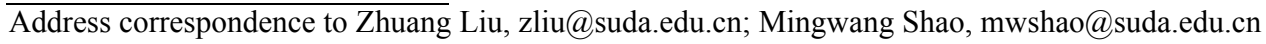


bioimaging [7-14]. Compared with traditional downconversion fluorescent dyes and QDs, near infrared (NIR)-to-visible upconversion optical bioprobes have a number of advantages. Autofluorescence is no longer an issue for upconversion luminescence (UCL) optical imaging, potentially allowing improved signalto-noise ratios and imaging sensitivity [15]. NIR irradiation with better tissue penetration ability is used as the excitation source, facilitating in vivo imaging. Moreover, UCNPs are resistant to photobleaching and have excellent photostability [16-18].

Recently, a number of groups have developed watersoluble UCNPs for in vitro imaging of cells $[15,19]$. Prasad et al. have reported in vivo whole body imaging of mice injected with UCNPs [20]. Using targeting ligand conjugated UCNPs, Li et al. have achieved efficient in vivo tumor targeting and UCL imaging [21, 22]. Although earlier work has shown that UCNPs with different $\mathrm{Ln}^{3+}$ dopants exhibit various colors [23, 24], multicolor-especially in vivo multicolorimaging using UCNPs has rarely been reported, except for a recent paper describing two-color imaging of mouse lymph nodes [25]. In this work, we have synthesized a series of UCNPs and functionalized them with polyethylene glycol (PEG) to confer water solubility and biocompatibility. The 'colors' of three types of UCNPs with different UCL emission spectra upon $980 \mathrm{~nm}$ laser excitation are readily distinguished by using a Maestro EX optical imaging system and spectral deconvolution. As a proof-of-principle experiment for in vivo multicolor imaging, we first imaged mice subcutaneously injected with UCNPs and then further used the nanoparticles for multiplexed lymph node imaging in vivo and ex vivo. Multicolor cell labeling by UCNPs and in vivo cell tracking using UCL imaging has also been realized. Furthermore, we compared the in vivo imaging sensitivities of QDsbased fluorescence imaging and UCNPs-based UCL imaging. We found the in vivo detection limit of UCNPs to be at least one order of magnitude lower than that of QDs in our current non-optimized imaging system. Our work shows the promise of the UCL imaging technique for future multiplexed biomedical imaging and diagnosis with high sensitivities.

\section{Experimental}

\subsection{Synthesis of $\mathrm{NaYF}_{4}: \mathrm{Yb}$, Er (Tm) nanoparticles}

The synthesis of $\mathrm{NaYF}_{4}: \mathrm{Yb}, \mathrm{Er}(\mathrm{Tm}) \mathrm{UCNPs}$ was carried out following a literature procedure with modifications [26]. All the syntheses were performed under standard oxygen-free conditions except where noted. $\mathrm{Y}_{2} \mathrm{O}_{3}, \mathrm{Yb}_{2} \mathrm{O}_{3}, \mathrm{Er}_{2} \mathrm{O}_{3}$, and $\mathrm{Tm}_{2} \mathrm{O}_{3}$ were purchased from Shanghai Chemical Industrial Co. and used as the starting materials without further purification. Lanthanide trifluoroacetates were prepared by dissolving the respective lanthanide oxides in trifluoroacetic acid $\left(\mathrm{CF}_{3} \mathrm{COOH}\right.$, Shanghai Chemical Industrial Co.). Oleic acid (OA, 90\%) and 1-octadecene (ODE > 90\%) were purchased from Sigma-Aldrich. Typically, $1 \mathrm{mmol}$ of $\mathrm{Ln}\left(\mathrm{CF}_{3} \mathrm{COO}\right)_{3}(\mathrm{UCNP} 1, \mathrm{Y}: \mathrm{Yb}: \mathrm{Er}=78 \%: 20 \%: 2 \%$; UCNP2, $\mathrm{Y}: \mathrm{Yb}: \mathrm{Tm}=78 \%: 20 \%: 2 \%$; and UCNP3, Y:Yb:Er $=69 \%$ : $30 \%: 1 \%), 20 \mathrm{mmol}$ of $\mathrm{NaF}$ and $20 \mathrm{~mL}$ of solvent $(10 \mathrm{~mL}$ $\mathrm{OA} / 10 \mathrm{~mL}$ ODE) were added simultaneously to a $100 \mathrm{~mL}$ three-necked flask and degassed at $100{ }^{\circ} \mathrm{C}$ for $1 \mathrm{~h}$ under vacuum. In the presence of nitrogen, the mixture was rapidly heated to $320^{\circ} \mathrm{C}$ and kept at this temperature for $30 \mathrm{~min}$ under vigorous magnetic stirring. After cooling to room temperature, the products were precipitated by addition of ethanol, separated by centrifugation, and washed repeatedly by ethanol and water. The resulting nanoparticles could be redispersed in various non-polar organic solvents.

\subsection{Synthesis of octylamine-poly(acrylic acid)- polyethylene glycol (OA-PAA-PEG) co-polymer}

Octylamine-poly(acrylic acid) (OA-PAA) co-polymer was synthesized following a literature protocol [27]. $3 \mathrm{mmol}$ of solid poly(acrylic acid) (PAA, $M \mathrm{w}=1800$, Sigma-Aldrich) and $1.5 \mathrm{mmol}$ of 1-ethyl-3-(3dimethylaminopropyl) carbodiimide hydrochloride) (EDAC, Sigma-Aldrich) were transferred into a $10 \mathrm{~mL}$ flask. $2 \mathrm{~mL}$ of dimethylformamide (DMF) was added to dissolve the mixture. About $1 \mathrm{mmol}$ of octylamine (Sigma-Aldrich) was added dropwise into the reaction flask. When the reaction was complete after $24 \mathrm{~h}$ at room temperature, the residue was acidified by $5 \%$ $\mathrm{HCl}$ to $\mathrm{pH} 2$. The precipitated product was separated by centrifugation $(8000 \mathrm{r} / \mathrm{min}$ for $5 \mathrm{~min}$ ), and then washed several times with water. The final product 
was lyophilized and stored below $-20^{\circ} \mathrm{C}$ until use.

$5.1 \mathrm{mg}$ of the synthesized OA-PAA $(\sim 0.01 \mathrm{mmol}$ leftover carboxyl acid) and $4 \mathrm{mg}$ of EDAC $(0.02 \mathrm{mmol})$ were dissolved in $2 \mathrm{~mL}$ of DMF in a $10 \mathrm{~mL}$ flask. $50 \mathrm{mg}$ of an amino-functionalized poly(ethylene glycol) PEG$5 \mathrm{~K}-\mathrm{NH}_{2}$ (0.01 mmol, PEG Bio Inc, Suzhou) dissolved in $1 \mathrm{~mL}$ of DMF was added into the reaction flask. The solution was stirred overnight. After transfer into water, the polymer solution was dialyzed against water using a 3500 Da cut-off membrane and then lyophilized. Successful PEGylation and octylamine conjugation were confirmed by the NMR spectrum of the final product.

\subsection{Functionalization of UCNPs with OA-PAA-PEG}

$500 \mu \mathrm{L}$ stock solutions of UCNPs were precipitated by centrifugation with the supernatant discarded. The nanoparticles were rinsed twice with ethanol and dispersed in $2 \mathrm{~mL}$ of chloroform. A second solution of $5 \mathrm{mg}$ of polymer in $2 \mathrm{~mL}$ chloroform was then added. After blow-drying the chloroform, the residue can be readily dissolved in water. The resultant solution was filtered through a $0.22-\mu \mathrm{m}$ syringe filter to remove large aggregates.

\subsection{Cell labeling and confocal microscope imaging}

KB cells (a human carcinoma cell line) were obtained from American Type Culture Collection (ATCC). All cell culture related reagents were purchased from Invitrogen. KB cells were cultured in RPMI-1640 medium supplemented with $10 \%$ fetal bovine serum (FBS) and 1\% penicillin/streptomycin. For confocal imaging, cells were seeded on a cover slide placed in a $5 \mathrm{~cm}$ plate and then incubated with $0.05 \mathrm{mg} / \mathrm{mL}$ of UCNP1 for $24 \mathrm{~h}$. After washing with phosphatebuffered saline (PBS), cells were fixed by $75 \%$ ethanol and imaged under a modified Leica SP3 confocal microscope. A $1 \mathrm{~W} 980 \mathrm{~nm}$ laser was introduced into the microscope as the excitation light. Both green $(\sim 550 \mathrm{~nm})$ and red UCL emissions ( 650 nm) of UCNP1 were recorded by the microscope.

\subsection{Animal experiments and in vivo imaging}

Balb/c mice ( $20 \mathrm{~g})$ and athymic nude mice $(\sim 20 \mathrm{~g})$ experiments (Suzhou Belda Bio-Pharmaceutical Co.) were performed under protocols approved by Soochow University Laboratory Animal Center.

For subcutaneous injection and UCL imaging, the OA-PAA-PEG-coated UCNP aqueous solutions $(15 \mu \mathrm{L}$, $1 \mathrm{mg} / \mathrm{mL}$ ) in $0.9 \% \mathrm{NaCl}$ saline solution were subcutaneously injected into the back area of nude mice $(\sim 20 \mathrm{~g})$. Mice were then imaged by a Maestro EX in vivo fluorescent imaging system using a $980 \mathrm{~nm}$ optical fiber-coupled laser as the excitation source. The laser power density was $\sim 0.2 \mathrm{~W} / \mathrm{cm}^{2}$ during imaging, which is a safe power according to previous in vivo photothermal therapy studies [28, 29]. An $850 \mathrm{~nm}$ shortpass emission filter was applied to prevent the interference of excitation light with the charge coupled device (CCD) camera. In vivo spectral imaging from $450 \mathrm{~nm}$ to $850 \mathrm{~nm}$ (in $10 \mathrm{~nm}$ steps) was carried out with an exposure time of $1000 \mathrm{~ms}$ for each image frame. Background was removed by using the spectral unmixing software. Mice were anaesthetized by isoflurane during imaging.

For lymph node mapping, OA-PAA-PEG-coated UCNPs $(10-20 \mu \mathrm{L})$ were intracutaneously injected into three different sites including the two paws and the chin of each mouse. Compared with semiconductor QDs used in previous work [30], the observed migration of the UCNPs appeared to be slower. This could be because the OA-PAA-PEG coating on UCNPs gives the nanoparticle surface protein resistance characteristics and reduces interactions of the UCNPs with lymph cells [31]. Two hours after injection, the mice were imaged by the Maestro system using $980 \mathrm{~nm}$ laser excitation with the injection sites shielded to avoid signal saturation on those sites during imaging.

For comparison of the sensitivities of QD fluorescence imaging and UCNP imaging, water soluble QD545 carboxyl semiconductor quantum dots $(8 \mu \mathrm{mol} / \mathrm{L}$ solution) and QD625 carboxyl semiconductor quantum dots $(8 \mu \mathrm{mol} / \mathrm{L}$ solution) were purchased from Invitrogen (USA) and Wuhua Jiayuan Quantum Dots Co. (China), respectively. The molar concentrations of QDs were determined by a well known literature method according to the product manuals [32]. The molecular mass of each UCNP was estimated by using the average nanoparticle diameter of $30 \mathrm{~nm}$ and the density of bulk $\mathrm{NaYF}_{4}\left(4.21 \mathrm{~g} / \mathrm{cm}^{3}\right)$ [33] as $3.58 \times$ $10^{7} \mathrm{~g} / \mathrm{mol}$. The molar concentrations of UCNPs were 
then calculated from their mass concentrations. Various concentrations of UCNP1 and two types of QDs were subcutaneously injected into mice for UCL and fluorescence imaging, respectively. $430-470 \mathrm{~nm}$ (blue) excitation and 510-550 nm (green) excitation were used for QD545 and QD625 imaging, respectively. The imaging exposure time was $3000 \mathrm{~ms}$ for UCNP1, $120 \mathrm{~ms}$ for QD545 and $160 \mathrm{~ms}$ for QD625. It should be noted that for QD fluorescence imaging, the autofluorescence saturated the CCD detector if too long an exposure time was used.

\subsection{In vivo cell tracking}

For cell labeling, $\sim 2$ million KB cells in each cell culture plate were incubated with UCNPs at a concentration of $\sim 0.05 \mathrm{mg} / \mathrm{mL}$ for $24 \mathrm{~h}$ at $37^{\circ} \mathrm{C}$. After extensively washing three times with serum-free RPMI-1640 medium, the cells were detached from the plate by trypsin-EDTA, resuspended in serum-free RMPI1640, and centrifuged to obtain cell pellets for UCL imaging. For in vivo imaging, three suspensions of $\mathrm{KB}$ cells ( $\sim$ million cells for each sample) after incubation with UCNP1, UCNP2, and UCNP3 were subcutaneously injected into mice, which were subsequently imaged by the UCL imaging system.

\subsection{Characterization}

The phase and crystallography of the products were characterized by using a Shimadzu XRD-6000 X-ray diffractometer with $\mathrm{Cu} \mathrm{K} \alpha$ radiation $(\lambda=0.15406 \mathrm{~nm})$. Scanning electron microscopy (SEM) images were obtained using a FEI Quanta 200F scanning electron microscope. Transmission electron microscopy (TEM) and high-resolution transmission electron microscopy (HRTEM) images of the nanocrystals were obtained using a Philips CM300 transmission electron microscope operating at an acceleration voltage of $200 \mathrm{kV}$. Fourier transform infrared (FTIR) absorption spectra of the products were recorded by using a ProStar LC240 IR spectrometer at $4 \mathrm{~cm}^{-1}$ resolution, over the wavenumber range $400-4000 \mathrm{~cm}^{-1}$. Thermogravimetricdifferential thermal analysis (TG-DTA) measurements of the products were performed using a Setaram TGA 92 instrument in the temperature range from room temperature to $750{ }^{\circ} \mathrm{C}$ at a heating rate of $10{ }^{\circ} \mathrm{C}$ $\mathrm{min}^{-1}$. Upconversion fluorescence spectra were obtained on a FluoroMax 4 luminescence spectrometer (HORIBA Jobin Yvon) with an external $980 \mathrm{~nm}$ laser diode (1 W, continuous wave with $1 \mathrm{~m}$ fibre, Beijing Hi-Tech Optoelectronics Co., Ltd) as the excitation source in place of the xenon lamp in the spectrometer.

\section{Results and discussion}

\subsection{Synthesis and functionalization of UCNPs}

A number of $\mathrm{NaYF}_{4}$ UCNPs doped with different lanthanide elements (UCNP1: $\mathrm{Y}: \mathrm{Yb}: \mathrm{Er}=78 \%: 20 \%: 2 \%$; UCNP2: Y:Yb:Tm = 78\%:20\%:2\%; UCNP3: Y:Yb:Er = 69\%:30\%:1\%) were synthesized following a literature procedure with slight modifications [26]. As-synthesized UCNPs coated by oleic acid were not soluble in water. A PEG and octylamine modified poly(acrylic acid) polymer (OA-PAA-PEG) was used to transfer the hydrophobic UCNPs into the aqueous phase (Fig. 1(a)). The coating of OA-PAA-PEG on UCNPs was formed

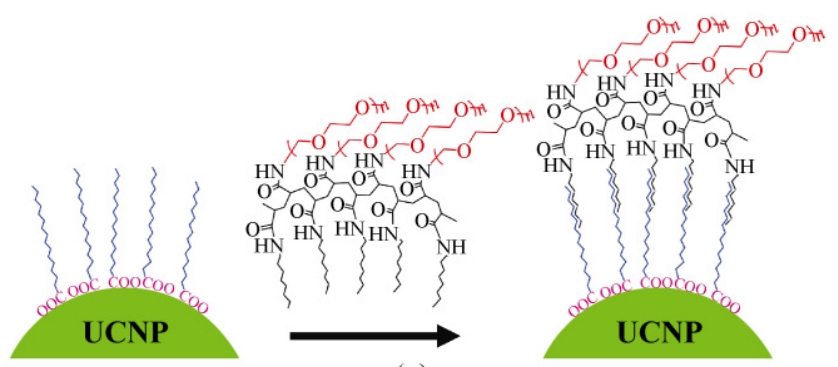

(a)

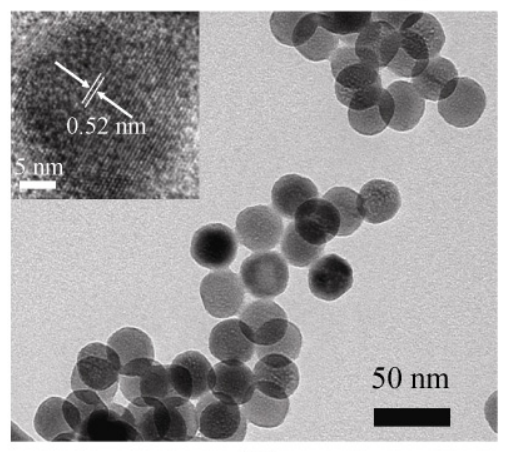

(b)

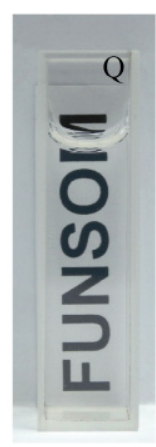

(c)

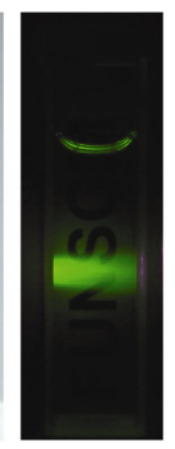

(d)
Figure 1 UCNPs functionalized with an amphiphilic polymer: (a) a schematic illustration of UCNPs with the OA-PAA-PEG polymer coating; (b) TEM images of the as-synthesized UCNP1 sample (the inset shows the HRTEM image of this sample); photos of the OA-PAA-PEG-coated UCNP1 sample in an aqueous solution under ambient light (c) and under $980 \mathrm{~nm}$ laser excitation (d)

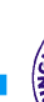

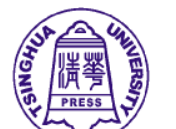

黑 Springer 
by hydrophobic interactions between hydrocarbon chains of the octylamine and the oleic acid. TEM images of the UCNP1 (Fig. 1(b)) revealed that the nanoparticles were monodisperse with an average diameter of $\sim 30 \mathrm{~nm}$. HRTEM (inset, Fig. 1(b)) showed lattice fringes with a $d$ spacing of $0.52 \mathrm{~nm}$, in good agreement with the lattice spacing of the (100) planes of hexagonal $\mathrm{NaYF}_{4}$ (Fig. S-1 in the Electronic Supplementary Material (ESM)). Similar electron microscopy results were also observed for other samples (UCNP2, UCNP3, Figs. S-2 and S-3 in the ESM). PEGylated UCNPs exhibited excellent water solubility (Figs. 1(c) and 1(d)). The successful PEGylation of UCNPs was also evidenced by IR spectroscopy and thermogravimetric analysis (Figs. S-4 and S-5 in the ESM). The hydrodynamic diameters of UCNP samples were measured to be $50-70 \mathrm{~nm}$ by dynamic light scattering (DLS) (Fig. S-6 in the ESM). The UCL emission spectra of three types of UCNPs in aqueous solutions were recorded by a fluorometer using a $980 \mathrm{~nm}$ laser as the excitation light (Fig. S-7 in the ESM).

\subsection{Multicolor UCL imaging}

Aqueous solutions of UCNP1, UCNP2, and UCNP3 were imaged by a Maestro in vivo spectral imaging system using a $980 \mathrm{~nm}$ optical fiber-coupled laser as the excitation source (Fig. S-8 in the ESM). An $850 \mathrm{~nm}$ short-pass filter was placed in front of the CCD camera (cooled to $0{ }^{\circ} \mathrm{C}$ ) to cut the excitation light. UCL emission spectra of three UCNP solutions (Fig. 2(a)) showed distinctive emission peak patterns. Different UCL 'colors' from the three types of UCNPs were easily differentiated in the image after spectral deconvolution without obvious cross-talk (Fig. 2(b), Fig. S-9 in the ESM). Green, red, and blue were artificially assigned to UCNP1, UCNP2, and UCNP3, respectively.

To demonstrate the feasibility of in vivo UCL multicolor imaging, we subcutaneously injected three different UCNP solutions and a solution of mixed UCNPs into the back of nude mice, and then imaged them using the Maestro system with the $980 \mathrm{~nm}$ laser as the excitation light (power density $\sim 0.2 \mathrm{~W} / \mathrm{cm}^{2}$ ). UCL emission spectra of three UCNPs solutions were used to unmix the obtained spectral image, showing clearly distinguished colors at their corresponding injection sites (Figs. 2(c)-2(g)). The co-localization of

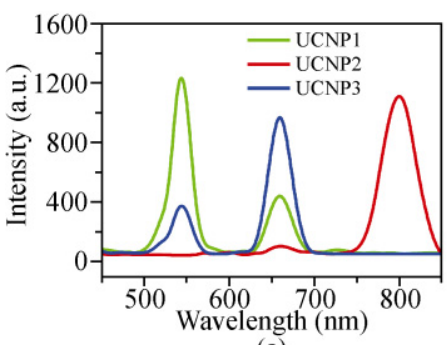

(a)

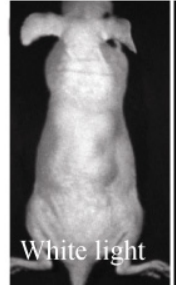

(c)

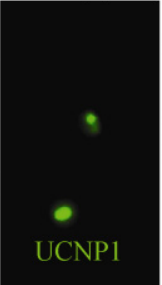

(d)

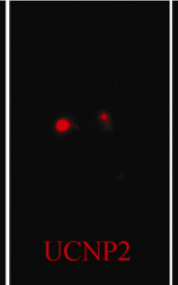

(e)

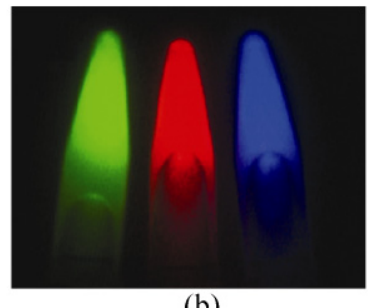

(b)
Figure 2 Multicolor UCL imaging: (a) UCL emission spectra of three UCNP solutions under $980 \mathrm{~nm}$ NIR laser excitation; (b) a multicolor fluorescence image of three UCNP solutions obtained by the Maestro in vivo imaging system (CRi, Inc.); (c) a white light image of a mouse subcutaneously injected with UCNPs; (d)-(f) in vivo multicolor images of a nude mouse subcutaneously injected with different UCNPs solutions; ( $g$ ) three colors of UCNPs were clearly differentiated after spectral unmixing

three colors at the point where the solution of mixed UCNPs was injected (top right) clearly revealed the ability of UCNPs to be used in multiplexed in vivo imaging (Fig. 2(g)).

\subsection{Multiplexed in vivo lymphatic imaging with UCNPs}

Local lymphatic drainage is an important route for the metastasis of cancer cells [34]. To this end, identification of the sentinel lymph nodes has become a common staging procedure for cancers which frequently metastasize to the local lymph nodes. However, evaluation of the lymphatic system is difficult due to its complicated small structures which are not directly accessible. Fluorescent QDs have been successfully employed for in vivo multicolor imaging of the lymphatic basins [30]. Although Kobayashi et al. have recently reported the use of two colors of UCNPs for lymph node mapping [25], simultaneous multicolor imaging of separated lymphatic basins (multiple lymphatic basins labeled with different colors) was not demonstrated. Here, we utilized three colors of UCNPs for multiplexed lymphangiography of three 
groups of lymph nodes. We simultaneously injected three solutions of OA-PAA-PEG coated UCNPs (10-20 $\mu \mathrm{L}$ of each) intracutaneously into three different sites including the two paws and the chin of each mouse (Fig. 3(a)), in order to monitor the lymphatic drainages in the neck and the upper trunk, where the most complicated lymphatic network exists in the body. The mice were again imaged by the Maestro system using the $980 \mathrm{~nm}$ laser excitation with the injection sites shielded during imaging. The primary lymph nodes to which each color of UCNPs migrated via lymphatic drainage were visualized by in vivo UCL imaging two hours after injection (Figs. 3(b) and 3(c)). The mice were dissected afterwards and imaged again. Six primary lymph nodes with two per color were clearly lit up (Figs. 3(d) and 3(e)). Our results show the capability of UCNPs in multicolor multiplexed lymph node mapping, which could provide a novel imaging method for future research on the lymphatic system.

\subsection{In vivo multicolor cell tracking with UCNPs}

In vivo cell tracking, especially stem cell tracking, is of great importance in biomedical research $[35,36]$. Here, we used UCNPs as a novel class of cell labeling agent for multicolor in vivo cell tracking and imaging.

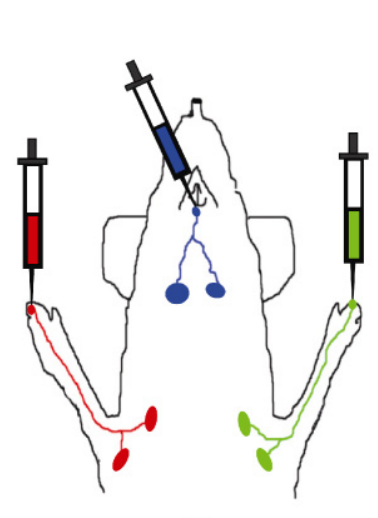

(a)
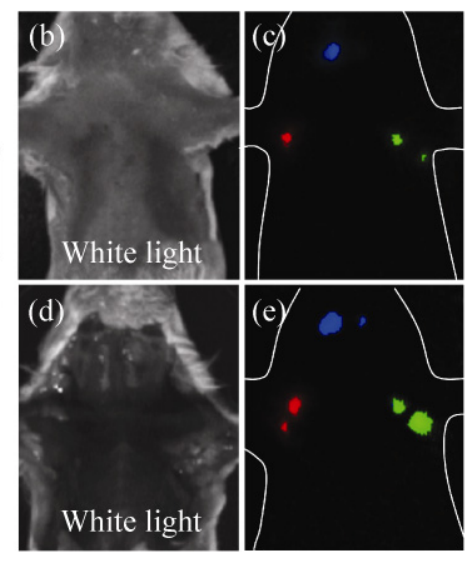

Figure 3 In vivo multiplexed lymphangiography with UCNPs: (a) a schematic illustration of UCNP-based lymph node mapping; (b) a white light image of a mouse injected with UCNPs at the sites indicated in (a); (c) a three-color spectrally resolved in vivo UCL image showing different UCNP colors from the corresponding lymph nodes under the skin; (d) a white light image of the same mouse after dissection; (e) a UCL image of the dissected mouse. The six primary lymph nodes are more clearly seen after the surgery
A human KB carcinoma cell line was used as the proof-of-concept model. KB cells were incubated with PEGylated UCNP1 at a concentration of $0.05 \mathrm{mg} / \mathrm{mL}$ for $24 \mathrm{~h}$, washed with PBS, and then fixed for confocal microscope imaging. A modified Leica laser-scanning confocal fluorescence microscope with a $980 \mathrm{~nm}$ laser as the excitation source was used to image the cell samples. Bright UCL signals were observed from cells incubated with UCNPs (Figs. 4(a) and 4(b)), displaying efficient uptake of UCNPs by the cells. In contrast, minimal background from the control untreated cells was observed (Fig. 4(c)). Standard in vitro cell toxicity tests revealed no obvious toxic effects of our PEGylated UCNPs on the treated cells (Fig. S-10 in the ESM).

To label cells with multiple colors of UCNPs, three plates of $\mathrm{KB}$ cells were incubated with solutions of UCNP1, UCNP2, and UCNP3 for $24 \mathrm{~h}$. The cells were washed extensively with serum-free cell medium, trypsinized, suspended in cell medium, and then
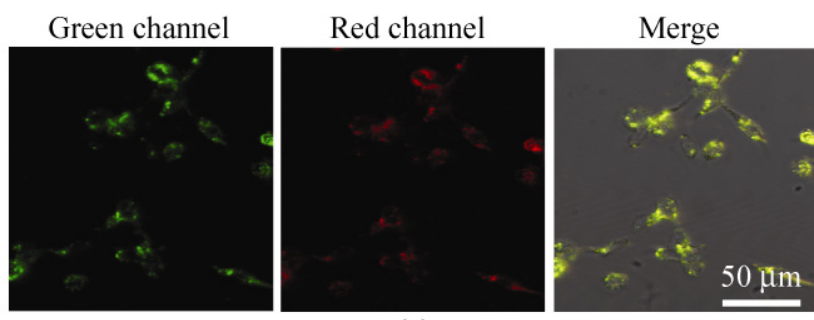

(a)
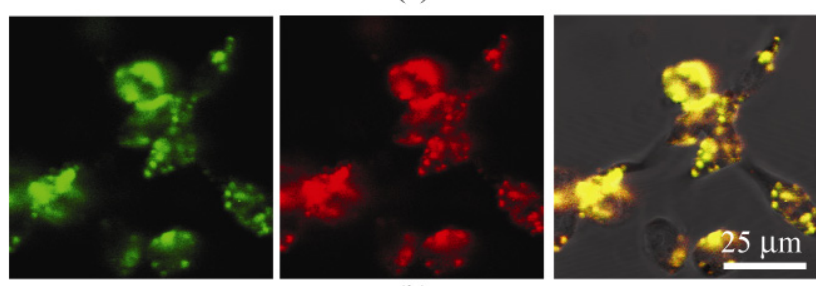

(b)
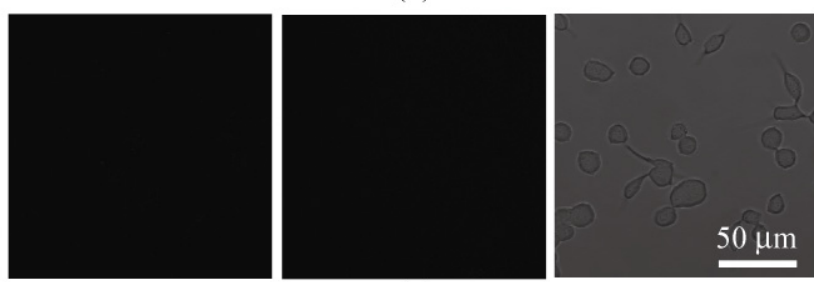

(c)

Figure 4 In vitro confocal UCL imaging of cells labeled by UCNPs. KB cells were incubated with UCNP1 before imaging. The green $(\sim 550 \mathrm{~nm})$ and red $(\sim 650 \mathrm{~nm})$ UCL emissions from UCNP1 under the $980 \mathrm{~nm}$ excitation were collected; (a) large area confocal UCL images of UCNP1 incubated cells; (b) high resolution confocal UCL images of UCNP1 incubated cells; (c) large area confocal UCL images of untreated control cells

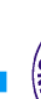


centrifuged to collect the cell pellets. UCL imaging revealed strong signals from the cell pellets without any background in the cell culture medium (Figs. 5(a) and 5(b)), suggesting efficient cellular uptake of UCNPs. Three cell pellets labeled by different UCNPs showed clearly differentiated 'colors' in the UCL image. The three cell suspensions were then subcutaneously injected into a mouse, which was immediately imaged by the modified Maestro system. UNCP-labeled cells were clearly visualized by multicolor UCL imaging in vivo (Figs. 5(c) and 5(d)). Tumors developed one week after inoculation of KB cancer cells as normal. UCL imaging of the mouse one week post KB cell injection revealed that UCNPs remained in the tumors (Figs. 5(e) and 5(f)). Our results demonstrate a novel way of in vivo multicolor cancer cell tracking with UCNPs.

Thus, we have, for the first time, achieved three-color multiplexed UCL imaging in vivo. More UCL colors may be obtained by varying the $\mathrm{Ln}^{3+}$ dopants in the $\mathrm{NaYF}_{4}$ UCNP system [23, 24]. The UCL emission
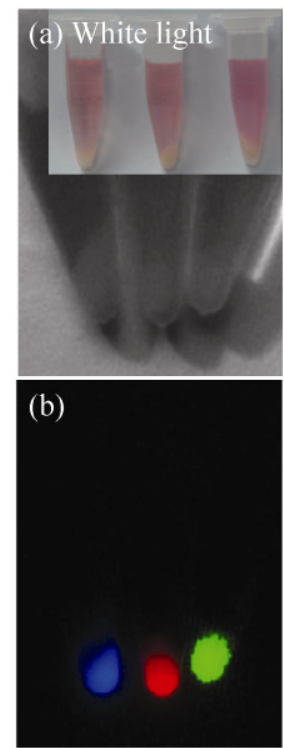
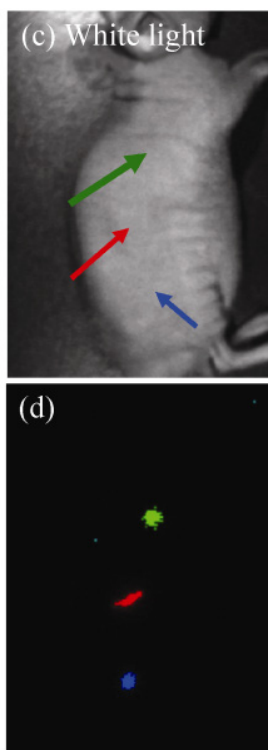
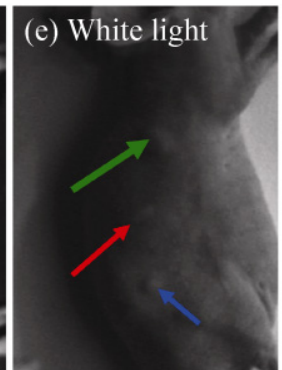

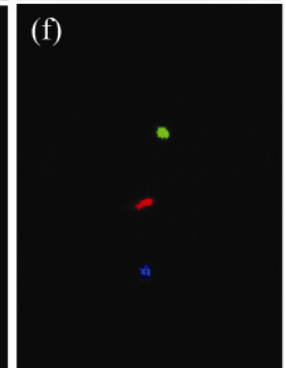

Figure 5 Multicolor in vivo cancer cell tracking and imaging. White light (a) and UCL (b) images of KB cell pellets after incubation with three colors of UCNPs for $24 \mathrm{~h}$. Excess UCNPs were completely removed by extensive washing. Substantial cellular uptake of UCNPs was evidenced by the bright UCL signals from the cell pellets. White light (c) and UCL (d) images of a nude mouse immediately after subcutaneous injection with three $\mathrm{KB}$ cell suspensions labeled by different colors of UCNPs. White light (e) and UCL (f) images of the same mouse one week after cancer cell injection. Three tumors were developed on the injected sites and clearly visualized by the three-color UCL imaging spectra could also be altered for further improved imaging multiplicity if UCNPs are coupled with QDs or fluorescent dyes for fluorescence resonance energy transfer (FRET) [37]. Much room remains for further development of multiplexed UCL imaging.

\subsection{Comparison of in vivo sensitivities of UCNP- based UCL imaging and QD-based fluorescence imaging}

Lastly, we compared the in vivo imaging sensitivity of UCNPs with that of commercial water-soluble semiconductor QDs. Mice subcutaneously injected with UCNP1 and two types of QDs at a series of concentrations were imaged by the Maestro system using different excitation sources. The molar concentrations of UCNPs were estimated using their average diameter and the density of $\mathrm{NaYF}_{4}$ (see Section 2.5 for details). While the UCNPs injected into mice at a concentration as low as $0.1 \mathrm{nmol} / \mathrm{L}$ were still detectable by UCL imaging (Figs. 6(a)-6(c)), QD545 and QD625 at concentrations of $5 \mathrm{nmol} / \mathrm{L}$ and below were barely visible due to the interference from mouse autofluorescence (Figs. 6(d)-6(i)). Because of the autofluorescence-free nature of UCL imaging, a long exposure time ( $\sim 3 \mathrm{~s} /$ frame) was employed in UCL imaging without introducing any obvious background (Fig. 6(c)), whereas for fluorescence imaging with QDs, the background fluorescence was substantial even for a short exposure (120-160 ms/frame) (Figs. 6(f) and 6(i)).

To quantify the detection limit of UCNPs and QDs, we plotted the signal intensities of UCNPs and QDs at their respective emission peaks against their concentrations (Fig. 7). An excellent linear relationship between UCL signal intensity and UCNP1 concentration was noted $\left(R^{2}=0.998\right)$ (Fig. 7(a)). The UCL imaging showed an ultra-low background (Fig. 7(b)), which was an order of magnitude lower than the detected UCL signals of UCNP1 at its lowest tested concentration $(0.1 \mathrm{nmol} / \mathrm{L})$. In marked contrast, the high and variable autofluorescence from the mouse body severely affected the detection sensitivity of QDs. The detected signals from $1.5 \mathrm{nmol} / \mathrm{L}$ QD545 and QD625 fell entirely into the autofluorescence ranges (Figs. 7(c) and 7(d)). We thus conclude that the detection limit of UCNP1 is at least one order of magnitude lower than that of QDs under our current in vivo imaging experimental conditions. 

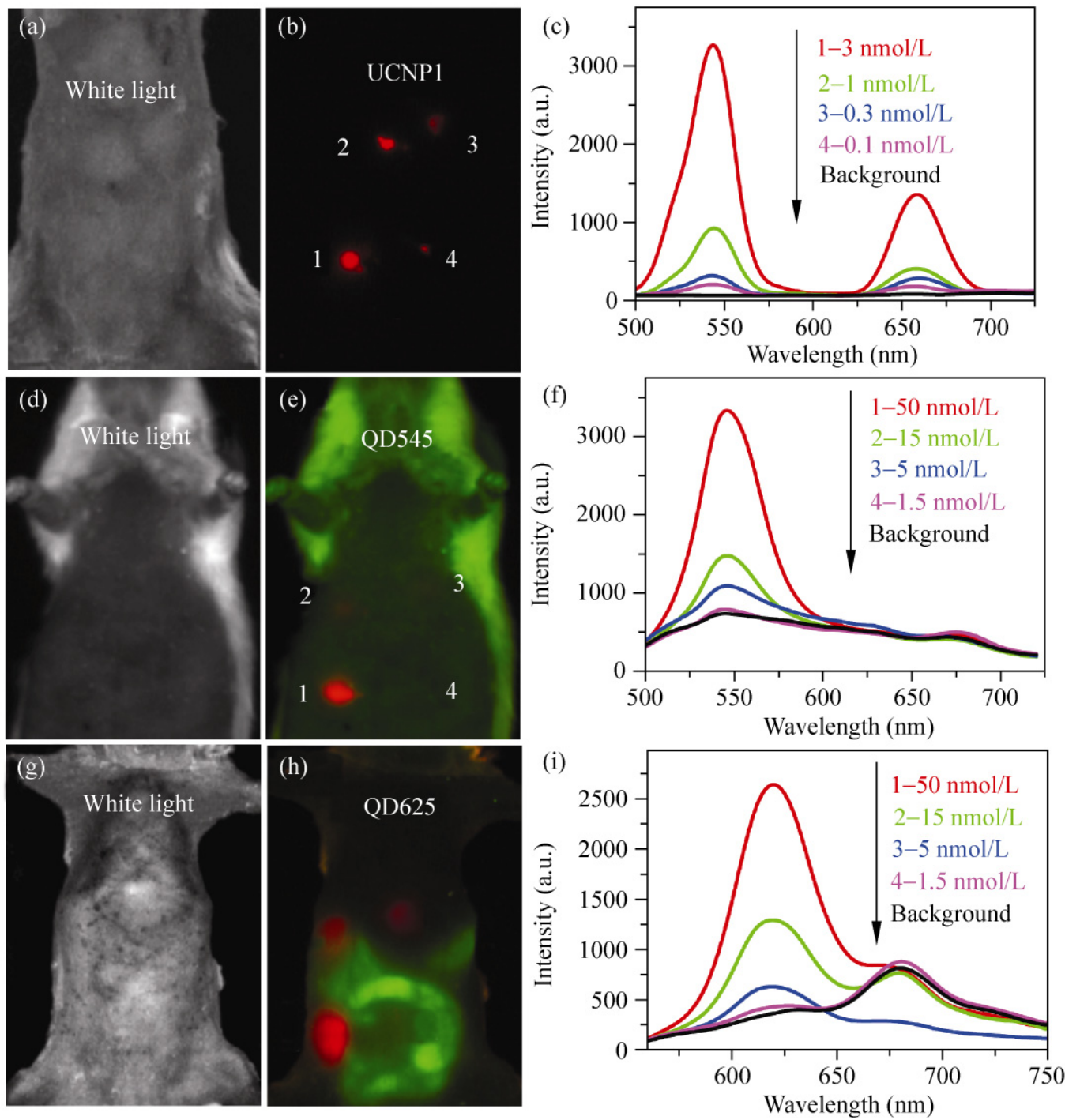

Figure 6 Comparison of imaging sensitivities between UCNPs and QDs: (a) a white light image of a mouse subcutaneously injected with various concentrations of UCNP1; (b) an in vivo UCL image of the injected mouse; (c) UCL emission spectra recorded at the injection sites; (d) and (g) white light images of mice subcutaneously injected with QDs; spectrally-resolved fluorescence images of QD545 injected mouse (e) and QD 625 injected mouse (h) (red and green colors represent QD fluorescence and autofluorescence, respectively); (f) and (i) fluorescence spectra recorded at the QD injection sites (the fluorescence spectra of $1.5 \mathrm{nmol} / \mathrm{L}$ QDs were nearly identical to the background spectra)

Although QDs have high quantum yields ( $40 \%$ for the water-soluble QDs used here), the detection sensitivity of traditional down-conversion fluorescence imaging is mainly limited by the autofluorescence background, which cannot be avoided by using higher excitation powers, longer exposure times or better CCD cameras as indicated in Figs. 6(f) and 6(i). Therefore, the in vivo detection limit of QDs observed in this work is almost the intrinsic limit of this technique and can barely be improved by better instrumentation. In contrast, for the autofluorescence-free UCL imaging, further enhanced sensitivity could readily be achieved if a highly sensitive deep-cold or electron multiplying (EM) CCD is used [21]. It is worth noting that the CRi Maestro system used in this work has among the best spectral unmixing functions, which is strongly beneficial for fluorescence imaging, but a less sensitive $\mathrm{CCD}$ camera $\left(0^{\circ} \mathrm{C}\right)$ than other mainstream in vivo optical imaging systems such as Xenogen IVIS (in which the CCD is cryogenically cooled to $-90{ }^{\circ} \mathrm{C}$ ) [38]. 


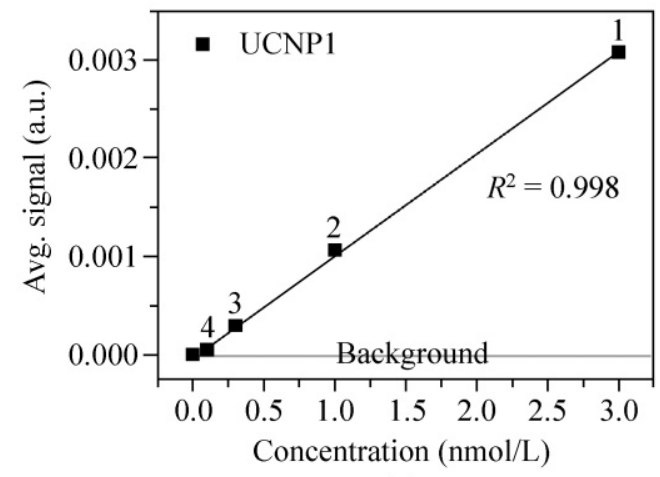

(a)

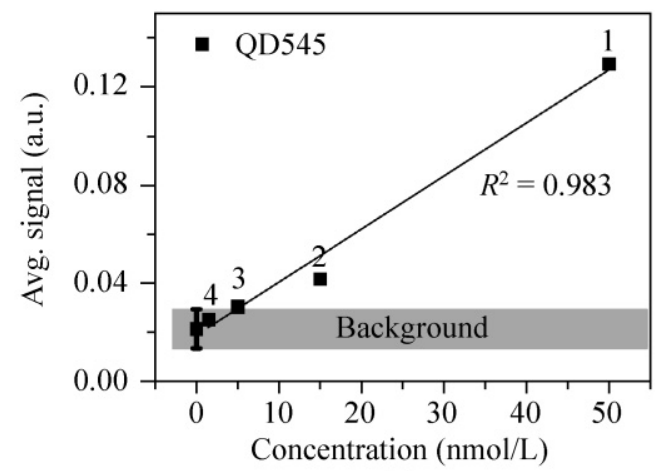

(c)

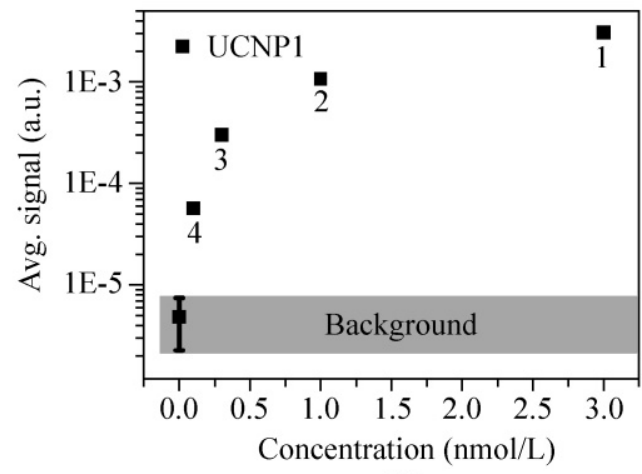

(b)

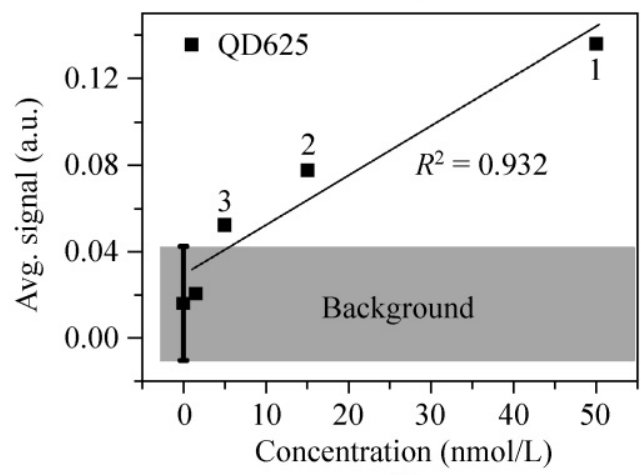

(d)

Figure 7 Quantitative comparison of in vivo detection sensitivities for UCNP1, QD545, and QD625. Concentration- dependent UCNP1 signals at the $540 \mathrm{~nm}$ emission with the $y$-axis plotted on a linear scale (a) and log scale (b). Concentration- dependent QD545 (c) and QD625 (d) emission signals at $550 \mathrm{~nm}$ and 620, respectively. The signal intensities from various concentrations of UCNPs and QDs were determined by averaging the region-of-interest (ROI) at their corresponding injection sites. The background signals from the mouse body without UCNP or QD injection were measured with error bars derived from standard deviations of four different non-injected regions on each mouse. The gray areas in (a), (c), and (d) represent the variation ranges of background signals

The advantage in the detection sensitivity of UCNPs over QDs is expected to be even more significant if an optimized optical imaging system (e.g., IVIS) is used. However, for applications such as real-time monitoring, where short exposure time is required during imaging, QD-based traditional fluorescence imaging will be much more favorable than UCL imaging relying on UCNPs. The two imaging techniques based on down-conversion and upconversion photoluminescence mechanisms are likely to be complementary for future optical imaging in biomedicine.

\section{Conclusion}

We have synthesized UCNPs with different emission colors and functionalized them with PEG to confer aqueous solubility. Multicolor in vivo UCL imaging has been realized by imaging subcutaneously injected
UCNPs and further demonstrated in multiplexed lymph node mapping and multicolor in vivo cancer cell tracking. Moreover, our results have shown that the autofluorescence-free UCL imaging exhibited significantly higher in vivo detection sensitivity than QD-based fluorescence imaging, highlighting the promise of UCL imaging in detecting molecular targets with low concentrations in biological systems. However, UCL biomedical imaging is still at its infant stage. The imaging sensitivity and multiplexity of UCNP-based UCL imaging could be further improved by enhancing the imaging instrumentation and optimizing UCNP materials. Future applications of this novel imaging methodology may also include multicolor multiplexed molecular imaging in vivo. UCL imaging is likely to be a useful supplement to current fluorescence imaging techniques since it can mitigate the various inherent limitations of the down- 
conversion imaging method. UCNPs have unique optical properties and great potential in highly sensitive multiplexed optical imaging, and may find many applications in future biomedical research.

\section{Acknowledgements}

This work was supported by the research start-up fund of Soochow University and the Research Grants Council of Hong Kong SAR (No. CityU5/CRF/08). We thank Dr. Yaping Du and Prof. Qiangbin Wang from Suzhou Institute of Nano-tech and Nano-bionics, Chinese Academy of Sciences, for helpful discussions and assistance in TEM characterization. The confocal microscope images were recorded with help from Leica Microsystems Ltd.

Electronic Supplementary Material: Supplementary information (various UCNP characterization data, illustration of spectral unmixing, and in vitro cellular toxicity data) are available in the online version of this article at http://dx.doi.org/10.1007/s12274-010-0036-2 and accessible free of charge.

Open Access: This article is distributed under the terms of the Creative Commons Attribution Noncommercial License which permits any noncommercial use, distribution, and reproduction in any medium, provided the original author(s) and source are credited.

\section{References}

[1] Gao, X.; Gao, Y.; Cui, Y.; Levenson, R. M.; Chung, L. W. $\mathrm{K}$; Nie, S. In vivo cancer targeting and imaging with semiconductor quantum dots. Nat. Biotechnol. 2004, 22, 969-976.

[2] Goldman, E. R.; Clapp, A. R.; Anderson, G. P.; Uyeda, H. T.; Mauro, J. M.; Medintz, I. L.; Mattoussi, H. Multiplexed toxin analysis using four colors of quantum dot fluororeagents. Anal. Chem. 2004, 76, 684-688.

[3] Liu, Z.; Li, X.; Tabakman, S. M.; Jiang, K.; Fan, S.; Dai, H. Multiplexed multi-color Raman imaging of live cells with isotopically modified single walled carbon nanotubes. $\mathrm{J}$. Am. Chem. Soc. 2008, 130, 13540-13541.

[4] Liu, Z.; Tabakman, S.; Sherlock, S.; Li, X.; Chen, Z.; Jiang, K.; Fan, S.; Dai, H. Multiplexed five-color molecular imaging of cancer cells and tumor tissues with carbon nanotube Raman tags in the near-infrared. Nano Res. 2010, 3, 222-223.

[5] Han, M.; Gao, X.; Su, J. Z.; Nie, S. Quantum-dot-tagged microbeads for multiplexed optical coding of biomolecules. Nat. Biotechnol. 2001, 19, 631-635.

[6] Li, X.; Wang, X.; Zhang, L.; Lee, S.; Dai, H. Chemically derived, ultrasmooth graphene nanoribbon semiconductors. Science 2008, 319, 1229-1232.

[7] Sandrock, T.; Scheife, H.; Heumann, E.; Hube, G. Highpower continuous-wave upconversion fiber laser at room temperature. Opt. Lett. 1997, 22, 808-810.

[8] Downing, E.; Hesselink, L.; Ralston, J.; Macfarlane, R. A three-color, solid-state, three-dimensional display. Science 1996, 273, 1185-1189.

[9] Kumar, R.; Nyk, M.; Ohulchanskyy, T. Y.; Flask, C. A.; Pras, P. N. Combined optical and MR bioimaging using rare earth ion doped $\mathrm{NaYF}_{4}$ nanocrystals. Adv. Funct. Mater. 2009, 19, 853-859.

[10] Wang, L.; Yan, R.; Huo, Z.; Wang, L.; Zeng, J.; Bao, J.; Wang, X.; Peng, Q.; Li, Y. Fluorescence resonant energy transfer biosensor based on upconversion-luminescent nanoparticles. Angew. Chem. Int. Ed. 2005, 44, 6054-6057.

[11] Yi, G.; Lu, H.; Zhao, S.; Ge, Y.; Yang, W.; Chen, D.; Guo, L. Synthesis, characterization, and biological application of size-controlled nanocrystalline $\mathrm{NaYF}_{4}$ : $\mathrm{Yb}$,Er infrared-to-visible up-conversion phosphors. Nano Lett. 2004, 4, 2191-2196.

[12] Mai, H.; Zhang, Y.; Si, R.; Yan, Z.; Sun, L.; You, L.; Yan, C. High-quality sodium rare-earth fluoride nanocrystals: Controlled synthesis and optical properties. J. Am. Chem. Soc. 2006, 128, 6426-6436.

[13] Wang, L. Y.; Zhang, Y.; Zhu, Y. Y. One-pot synthesis and strong near-infrared upconversion luminescence of poly(acrylic acid)-functionalized $\mathrm{YF}_{3}: \mathrm{Yb}^{3+} / \mathrm{Er}^{3+}$ nanocrystals. Nano Res. 2010, 3, 317-325.

[14] Wang, F.; Han, Y.; Lim, C. S.; Lu, Y. H.; Wang, J.; Xu, J.; Chen, H. Y.; Zhang, C.; Hong, M. H.; Liu, X. G. Simultaneous phase and size control of upconversion nanocrystals through lanthanide doping. Nature 2010, 463, 1061-1065.

[15] Yu, M. X.; Li, F. Y.; Chen, Z. G.; Hu, H.; Zhan, C.; Yang, H.; Huang, C. H. Laser scanning up-conversion luminescence microscopy for imaging cells labeled with rare-earth nanophosphors. Anal. Chem. 2009, 81, 930-935.

[16] Waynant, R. W.; Ilev, I. K.; Gannot, I. Mid-infrared laser applications in medicine and biology. Philos. Trans. R. Soc. London Ser. A 2001, 359, 635-644.

[17] Yi, G. S.; Chow, G. M. Synthesis of hexagonal-phase $\mathrm{NaYF}_{4}: \mathrm{Yb}, \mathrm{Er}$ and $\mathrm{NaYF}_{4}: \mathrm{Yb}, \mathrm{Tm}$ nanocrystals with efficient up-conversion fluorescence. Adv. Funct. Mater. 2006, 16, 2324-2329.

[18] Mai, H.; Zhang, Y.; Sun, L.; Yan, C. Highly efficient multicolor up-conversion emissions and their mechanisms 
of monodisperse $\mathrm{NaYF}_{4}: \mathrm{Yb}, \mathrm{Er}$ core and core/shell-structured nanocrystals. J. Phys. Chem. C 2007, 111, 13721-13729.

[19] Jalil, R. A.; Zhang, Y. Biocompatibility of silica coated $\mathrm{NaYF}_{4}$ upconversion fluorescent nanocrystals. Biomaterials 2008, 29, 4122-4128.

[20] Nyk, M.; Kumar, R.; Ohulchanskyy, T. Y.; Bergey, E. J.; Prasad, P. N. High contrast in vitro and in vivo photoluminescence bioimaging using near infrared to near infrared up-conversion in $\mathrm{Tm}^{3+}$ and $\mathrm{Yb}^{3+}$ doped fluoride nanophosphors. Nano Lett. 2008, 8, 3834-3838.

[21] Xiong, L.; Chen, Z.; Tian, Q.; Cao, T.; Xu, C.; Li, F. High contrast upconversion luminescence targeted imaging in vivo using peptide-labeled nanophosphors. Anal. Chem. 2009, 81, 8687-8694.

[22] Xiong, L. Q.; Chen, Z. G.; Yu, M. X.; Li, F. Y.; Liu, C.; Huang, C. H. Synthesis, characterization, and in vivo targeted imaging of amine-functionalized rare-earth up-converting nanophosphors. Biomaterials 2009, 30, 5592-5600.

[23] Wang, F.; Liu, X. G. Upconversion multicolor fine-tuning: Visible to near-infrared emission from lanthanide-doped $\mathrm{NaYF}_{4}$ nanoparticles. J. Am. Chem. Soc. 2008, 130, 5642-5643.

[24] Yin, A. X.; Zhang, Y. W.; Sun, L. D.; Yan, C. H. Colloidal synthesis and blue based multicolor upconversion emissions of size and composition controlled monodisperse hexagonal $\mathrm{NaYF}_{4}$ : Yb,Tm nanocrystals. Nanoscale 2010, 2, 953-959.

[25] Kobayashi, H.; Kosaka, N.; Ogawa, M.; Morgan, N. Y.; Smith, P. D.; Murray, C. B.; Ye, X.; Collins, J.; Kumar, G. A.; Bell, H.; Choyke, P. L. In vivo multiple color lymphatic imaging using upconverting nanocrystals. J. Mater. Chem. 2009, 19, 6481-6484.

[26] Liu, C.; Wang, H.; Li, X.; Chen, D. Monodisperse, sizetunable and highly efficient $\beta$ - $\mathrm{NaYF}_{4}: \mathrm{Yb}, \mathrm{Er}(\mathrm{Tm})$ upconversion luminescent nanospheres: Controllable synthesis and their surface modifications. J. Mater. Chem. 2009, 19, 3546-3553.

[27] Zhou, M.; Nakatani, E.; Gronenberg, L. S.; Tokimoto, T.; Wirth, M. J.; Hruby, V. J.; Roberts, A.; Lynch, R. M.; Ghosh, I. Peptide-labeled quantum dots for imaging GPCRs in whole cells and as single molecules. Bioconjugate Chem. 2007, 18, 323-332.

[28] von Maltzahn, G.; Park, J. H.; Agrawal, A.; Bandaru, N. K.; Das, S. K.; Sailor, M. J.; Bhatia, S. N. Computationally guided photothermal tumor therapy using long-circulating gold nanorod antennas. Cancer Res. 2009, 69, 3892-3900.

[29] Moon, H. K.; Lee, S. H.; Choi, H. C. In vivo near-infrared mediated tumor destruction by photothermal effect of carbon nanotubes. ACS Nano 2009, 3, 3707-3713.

[30] Kobayashi, H.; Hama, Y.; Koyama, Y.; Barrett, T.; Regino, C. A. S.; Urano, Y.; Choyke, P. L. Simultaneous multicolor imaging of five different lymphatic basins using quantum dots. Nano Lett. 2007, 7, 1711-1716.

[31] Yang, S.; Cao, L.; Luo, P. G.; Lu, F.; Wang, X.; Wang, H.; Meziani, M. J.; Liu, Y.; Qi, G.; Sun, Y. Carbon dots for optical imaging in vivo. J. Am. Chem. Soc. 2009, 131, 11308-11309.

[32] Yu, W. W.; Qu, L.; Guo, W.; Peng, X. Experimental determination of the extinction coefficient of CdTe, CdSe, and CdS nanocrystals. Chem. Mater. 2003, 15, 2854-2860.

[33] Pyatenko, Y. A.; Voronkov, A. A. The formula of gagarinite. J. Struct. Chem. 1962, 3, 696-697.

[34] Kobayashi, H.; Koyama, Y.; Barrett, T.; Hama, Y.; Regino, C. A. S.; Shin, I. S.; Jang, B. S.; Le, N.; Paik, C. H.; Choyke, P. L.; Urano, Y. Multimodal nanoprobes for radionuclide and five-color near-infrared optical lymphatic imaging. ACS Nano 2007, 1, 258-264.

[35] Lo Celso, C.; Fleming, H. E.; Wu, J. W.; Zhao, C. X.; Miake-Lye, S.; Fujisaki, J.; Cote, D.; Rowe, D. W.; Lin, C. P.; Scadden, D. T. Live-animal tracking of individual haematopoietic stem/progenitor cells in their niche. Nature 2009, 457, 92-96.

[36] Schroeder, T. Imaging stem-cell-driven regeneration in mammals. Nature 2008, 453, 345-351.

[37] Li, Z. Q.; Zhang, Y.; Jiang, S. Multicolor core/shell-structured upconversion fluorescent nanoparticles. Adv. Mater. 2008, 20, 4765-4769.

[38] See: http://www.caliperls.com/assets/011/6716.pdf 